\title{
ON THE DETERMINANT AND THE HOLONOMY OF EQUIVARIANT ELLIPTIC OPERATORS
}

\author{
KENJI TSUBOI
}

(Communicated by Ronald Stern)

\begin{abstract}
Let $M$ be a closed oriented smooth manifold, $G$ a compact Lie group consisting of diffeomorphisms of $M, P \rightarrow Z$ a principal $G$-bundle with a connection and $D$ a $G$-equivariant elliptic operator. Then a locally constant family of elliptic operators and its determinant line bundle over $Z$ are naturally defined by $D$. Moreover the holonomy of the determinant line bundle is defined by the connection in $P$. In this note, we give an explicit formula to calculate the holonomy (Theorem 1.4) and give a proof of the Witten holonomy formula (Theorem 1.7) in the special case above.
\end{abstract}

\section{Main Results}

Let $M$ be a closed oriented smooth manifold, $G$ a compact Lie group consisting of diffeomorphisms of $M, P \rightarrow Z$ a principal $G$-bundle over a smooth manifold $Z$ with a connection and $D$ a $G$-equivariant elliptic operator. Then, for any $g \in G$, the index of $D$ evaluated at $g$, Index $(D, g)$, is defined by

$$
\operatorname{Index}(D, g)=\operatorname{tr}\left(\left.g\right|_{\text {ker } D}\right)-\operatorname{tr}\left(\left.g\right|_{\text {coker } D}\right)
$$

and can be calculated by the well-known fixed point formula (cf. [1], [2] or [5]). On the other hand, the determinant of $D$ evaluated at $g, \operatorname{det}(D, g)$, is defined by

$$
\operatorname{det}(D, g)=\operatorname{det}\left(\left.g\right|_{\text {ker } D}\right) / \operatorname{det}\left(\left.g\right|_{\text {coker } D}\right) .
$$

Then the next proposition is an immediate consequence of the elementary result of Lemma 1 in Appendix.

Proposition 1.1. Let $g \in G$ be any element of finite order $p$. Then the next equality holds:

$$
\operatorname{det}(D, g)=\exp \frac{2 \pi i}{p} \sum_{k=1}^{p-1} \frac{1}{1-e^{-2 \pi i k / p}}\left\{\operatorname{Index}(D)-\operatorname{Index}\left(D, g^{k}\right)\right\}
$$

where $\operatorname{Index}(D)=\operatorname{Index}(D, 1)$ is the numerical index of $D$.

Received by the editors November 2, 1993.

1991 Mathematics Subject Classification. Primary 58G26; Secondary 58G10.

Key words and phrases. The determinant and the holonomy of elliptic operators.

The author is grateful to Professor Futaki for very useful discussions. 
Remark 1.2. The homomorphism $\operatorname{det}(D, \cdot): G \rightarrow S^{1}$ defined by $\operatorname{det}(D, g)$ is determined by its restriction to the dense subset of $G$ which consists of all elements of finite order.

Now we assume that $M$ is a $2 n$-dimensional closed Riemannian manifold with a Spin $^{c}$-structure and a connection in the associated $S^{1}$-bundle of the Spin $^{c}$-structure. We assume that $G$ acts on $M$ as isometries and that the action of $G$ preserves the $\operatorname{Spin}^{c}$-structure and the $S^{1}$-connection. Let $E$ be a hermitian vector bundle (or virtual vector bundle) over $M$ with a unitary connection. We assume that the action of $G$ lifts to a connection-preserving unitary action on $E$. Then we can define the $G$-equivariant $\operatorname{Spin}^{c}$-Dirac operator $D$ on $M$

$$
D: \Gamma\left(S^{+} \otimes E\right) \rightarrow \Gamma\left(S^{-} \otimes E\right)
$$

(for the definition of the half spinor bundles $S^{ \pm}$, see [7, pp. 106-108]) and a line bundle $\operatorname{det}(D)$ over $Z$ by

$$
\operatorname{det}(D)=P \times_{G}\left(\left(\wedge^{r} \operatorname{ker} D\right)^{*} \otimes\left(\wedge^{s} \operatorname{coker} D\right)\right)
$$

where $r, s$ denote the dimensions of the finite-dimensional (complex) $G$ modules $\operatorname{ker} D, \operatorname{coker} D$. Note that the $G$-equivariant elliptic operator $D$ naturally defines a locally constant elliptic family $P \times{ }_{G} D$ parametrized by $Z$ and the determinant line bundle defined by this elliptic family is isomorphic to $\operatorname{det}(D)$ above (see [6, pp. 133-134]). The connection in $P$ naturally defines a connection in $\operatorname{det}(D)$ and we can regard $\operatorname{det}(D)$ as a line bundle with a connection. On the other hand, for any $g \in G$, by considering the mapping torus

$$
M_{g}=M \times[0,1] / \sim \quad \text { where }(m, 0) \sim(g(m), 1),
$$

we can also define a locally constant family of Dirac operators parametrized by $S^{1}$. Here the horizontal subspaces of the fibration $M_{g} \rightarrow S^{1}$ is given by the $[0,1]$-directed vectors. Then we can define as in [7] the determinant line bundle $L(g)$ over $S^{1}$. Note that, if a horizontal lift $\tilde{\gamma}$ of an oriented loop $\gamma$ in $Z$ connects any fixed base point $b$ in $P$ with $b \cdot g^{-1}$, it is not difficult to see that $L(g)$ is isomorphic to the restriction of $\operatorname{det}(D)$ to the loop $\gamma$ as a line bundle with a connection. Now it can be seen that the holonomy of $L(g)$ around $S^{1}$, which we denote by $\operatorname{hol}(D, g)$, is equal to $\operatorname{det}(D, g)$ and hence the next theorem follows immediately from Proposition 1.1.

Theorm 1.4. Let $g \in G$ be any element of finite order $p$. Then we have

$$
\operatorname{hol}(D, g)=\exp \frac{2 \pi i}{p} \sum_{k=1}^{p-1} \frac{1}{1-e^{-2 \pi i k / p}}\left\{\operatorname{Index}(D)-\operatorname{Index}\left(D, g^{k}\right)\right\},
$$

and hence $\operatorname{hol}(D, g)$ is calculated explicitly by using the Atiyah-Bott-Singer fixed point formula.

Now the tangent bundle of $M_{g}$ splits as the direct sum of the tangent bundle of $M$ and the trivial real line bundle defined by [0,1]-directed vectors. Hence the Riemannian metric and the $\mathrm{Spin}^{c}$-structure on $M_{g}$ are naturally defined by those on $M$ together with the standard metric and the trivial $\operatorname{Spin}^{c}$-structure on $[0,1]$. Moreover the associated $S^{1}$-bundle of the $\operatorname{Spin}^{c}$-structure over $M_{g}$ and its connection are naturally defined by the $S^{1}$-bundle over $M$ and the 
standard globally flat connection in the [0,1]-directed trivial real line bundle. Let $S_{g}$ be the spinor bundle with respect to the above Spinc ${ }^{c}$-structure on $M_{g}$ and $E_{g}$ the hermitian vector bundle (or virtual vector bundle) over $M_{g}$ with a unitary connection defined by the mapping torus construction (1.3). Then the Spin $^{c}$-Dirac operator

$$
A_{g}: \Gamma\left(S_{g} \otimes E_{g}\right) \rightarrow \Gamma\left(S_{g} \otimes E_{g}\right)
$$

is defined and

$$
\xi_{g}=\frac{1}{2}\left(\eta_{g}+\operatorname{dim} \operatorname{ker} A_{g}\right)
$$

is defined by the eta invariant $\eta_{g}$ of $A_{g}$. Note that, using the same argument as in [4], we can see that $\xi_{g}$ modulo integer is continuous in $g$.

Now we assume that $g \in G$ has a finite order $p$. Let $X=M \times D^{2}$, $Y=\partial X=M \times S^{1}$ be the product Riemannian Spin $^{c}$-manifolds with the Spin $^{c}$-structures induced from the Spin ${ }^{c}$-structure on $M$ and the trivial Spin ${ }^{c}$ structures on $D^{2}, S^{1}$. We give the metric $p^{2} d s^{2}$ on $S^{1}$ where $d s^{2}$ is the standard metric on $S^{1}$ and a rotationally symmetric Riemannian metric on $D^{2}$ which is the product metric near $\partial D^{2}=S^{1}$. The Levi-Civita connection of this metric defines the connection in the associated $S^{1}$-bundles over $D^{2}, S^{1}$. Then we can define the actions of $\mathbb{Z}_{p}=\langle g\rangle$ on $X$ and on $\partial X=Y$ as follows:

$$
g \cdot\left(m, r e^{i \theta}\right)=\left(g(m), r e^{i \theta+2 \pi i / p}\right)
$$

for $\left(m, r e^{i \theta}\right) \in X=M \times D^{2} ; 0 \leq r \leq \frac{p}{2 \pi}, 0 \leq \theta \leq 2 \pi$. Note that $\mathbb{Z}_{p}$ acts freely on $Y$ and $Y / \mathbb{Z}_{p}$ is equal to $M_{g}$. Let $q_{X}: X=M \times D^{2} \rightarrow M$, $q_{Y}: Y=M \times S^{1} \rightarrow M$ be projections, and let $E_{X}, E_{Y}$ denote the hermitian vector bundles (or virtual vector bundles) $q_{X}^{*} E=E \times D^{2}, q_{Y}^{*} E=E \times S^{1}$ on $X, Y$ provided with naturally induced unitary connections. Let

$$
\begin{aligned}
& B: \Gamma\left(S_{X}^{+} \otimes E_{X}\right) \rightarrow \Gamma\left(S_{X}^{-} \otimes E_{X}\right), \\
& A: \Gamma\left(S_{Y} \otimes E_{Y}\right) \rightarrow \Gamma\left(S_{Y} \otimes E_{Y}\right)
\end{aligned}
$$

be the $\mathbb{Z}_{p}$-equivariant Spin $^{c}$-Dirac operators on $X, Y$ where $S_{X}^{ \pm}, S_{Y}$ are the spinor bundles over $X, Y$ with respect to the $\operatorname{Spin}^{c}$-structures on $X$, $Y$ respectively. Then it is clear that the spinor bundle $S_{g}$ is equal to the spinor bundle with respect to the Spin ${ }^{c}$-structure on $M_{g}$ induced from the $\mathbb{Z}_{p^{-}}$ invariant $\operatorname{Spin}^{c}$-structure on $Y$. Moreover it is also clear that $E_{g}$ is equal to the quotient $E_{Y} / \mathbb{Z}_{p}$ and that the Spinc-Dirac operator $A_{g}$ on $M_{g}$ is equal to the quotient $A / \mathbb{Z}_{p}$. Here we have the following:

Proposition 1.6. Let $g \in G$ be any element of finite order $p$. Then we have the right-hand side of $(1.5)=(-1)^{\operatorname{Index}(D)} e^{-2 \pi i \xi_{g}}$.

Proof. For any $h \in \mathbb{Z}_{p}$, let $\eta_{Y}(h)$ denote the eta invariant of $A$ evaluated at $h$ (cf. [4]). Then it follows from the same arguments as in [8] that

$$
\xi_{g}=\frac{1}{2}\left(\eta_{g}+\operatorname{dim} \operatorname{ker} A_{g}\right)=\frac{1}{p} \sum_{k=1}^{p}\left(\frac{1}{2} \eta_{Y}\left(g^{k}\right)+\frac{1}{2} \operatorname{tr}\left(\left.g^{k}\right|_{\text {ker } A}\right)\right) .
$$

On the other hand, it follows from Theorem 1.2 in [8] that

$$
\frac{1}{2} \eta_{Y}\left(g^{k}\right)+\frac{1}{2} \operatorname{tr}\left(\left.g^{k}\right|_{\operatorname{ker} A}\right)+\operatorname{Index}\left(B, g^{k}\right)
$$


is equal to the integral

$$
\int_{X} \operatorname{ch}\left(E_{X}\right) \exp \frac{\mathrm{c}_{1}(S, X)}{2} \widehat{A}(X)
$$

if $k=p$, and is equal to the summation of certain characteristic numbers $\mathfrak{A}[N]$

$$
\sum_{N \subset \Omega(X)} \mathfrak{A}[N]
$$

if $k \neq p$, where $\operatorname{Index}\left(B, g^{k}\right)$ is the $g^{k}$-index (i.e., the index evaluated at $g^{k}$ ) of $B$ with the global boundary condition considered in Theorem (3.10) in [3], $\operatorname{ch}\left(E_{X}\right)$ is the Chern character form of $E_{X}, \mathrm{c}_{1}(S, X)$ is the first Chern form of the associated $S^{1}$-bundle of the $\operatorname{Spin}^{c}$-structure on $X$ with respect to the $S^{1}$ connection, $\widehat{A}(X)$ is the total $\widehat{A}$-form of $T X$ and $\Omega(X)$ is the fixed point set of the $g^{k}$-action $(k \neq p)$ on $X$ consisting of closed connected submanifolds $N$. Now it is easy to see that the fixed point set $\Omega(X)$ coincides with the fixed point set $\Omega(M)$ of the $g^{k}$-action on $M=M \times\{0\} \subset M \times D^{2}=X$ and the normal bundles $\nu(N, X)$ of $N$ in $X$ is isomorphic to the direct sum of the normal bundles $\nu(N, M)$ of $N$ in $M$ and the trivial bundles $N \times \mathbb{R}^{2}$. Here $g$ acts on $N \times \mathbb{R}^{2}$ via the $2 \pi / p$-rotation of the fiber $\mathbb{R}^{2}$. Hence, considering the fixed point formula (cf. [5]), we can see that the quantity $\sum_{N \subset \Omega(X)} \mathfrak{A}[N]$ is related to the index of the operator $D$ on $M$ as follows:

$$
\sum_{N \subset \Omega(X)} \mathfrak{A}[N]=\frac{1}{1-e^{-2 \pi i k / p}} \operatorname{Index}\left(D, g^{k}\right) .
$$

On the other hand, it is clear that

$$
\mathrm{c}_{1}(S, X)=q_{X}^{*} \mathrm{c}_{1}(S, M)+q_{D}^{*} \mathrm{c}_{1}\left(D^{2}\right)
$$

where $\mathrm{c}_{1}(S, M)$ is the first Chern form of the associated $S^{1}$-bundle of the Spin ${ }^{c}$-structure on $M$ with respect to the $S^{1}$-connection, $q_{D}: X=M \times D^{2} \rightarrow$ $D^{2}$ is the projection and $c_{1}\left(D^{2}\right)$ is the first Chern form of $D^{2}$ with respect to the $S^{1}$-connection which is rotationally symmetric and is product near the boundary. Moreover, since

$$
\operatorname{ch}\left(E_{X}\right)=q_{X}^{*} \operatorname{ch}(E), \quad \widehat{A}(X)=q_{X}^{*} \widehat{A}(M)
$$

and

it follows that

$$
\int_{D^{2}} \exp \frac{\mathrm{c}_{1}\left(D^{2}\right)}{2}=\int_{D^{2}} \frac{\mathrm{c}_{1}\left(D^{2}\right)}{2}=\frac{1}{2}
$$

$\int_{X} \operatorname{ch}\left(E_{X}\right) \exp \frac{\mathrm{c}_{1}(S, X)}{2} \widehat{A}(X)=\frac{1}{2} \int_{M} \operatorname{ch}(E) \exp \frac{\mathrm{c}_{1}(S, M)}{2} \widehat{A}(M)=\frac{1}{2} \operatorname{Index}(D)$.

Hence we can deduce the following equality.

$$
\xi_{g}=\frac{1}{p} \sum_{k=1}^{p-1} \frac{1}{1-e^{-2 \pi i k / p}} \operatorname{Index}\left(D, g^{k}\right)+\frac{1}{2 p} \operatorname{Index}(D)-\frac{1}{p} \sum_{k=1}^{p} \operatorname{Index}\left(B, g^{k}\right) \text {. }
$$

Now it follows from Lemma 2 in Appendix that

$$
\frac{1}{p} \sum_{k=1}^{p} \operatorname{Index}\left(B, g^{k}\right)=0 \quad \bmod . \mathbb{Z}
$$


and from Lemma 3 in Appendix that

$$
\frac{1}{2 p} \operatorname{Index}(D)=\frac{1}{2} \operatorname{Index}(D)-\frac{1}{p} \sum_{k=1}^{p-1} \frac{1}{1-e^{-2 \pi i k / p}} \operatorname{Index}(D) .
$$

Thus we can conclude that

$$
e^{-2 \pi i \xi_{g}}=(-1)^{\operatorname{Index}(D)} \exp \frac{2 \pi i}{p} \sum_{k=1}^{p-1} \frac{1}{1-e^{-2 \pi i k / p}}\left\{\operatorname{Index}(D)-\operatorname{Index}\left(D, g^{k}\right)\right\} .
$$

This completes the proof.

Since both $\operatorname{hol}(D, g)(=\operatorname{det}(D, g))$ and $e^{-2 \pi i \xi_{g}}$ are continuous in $g$, it follows from Remark 1.2, Theorem 1.4 and Proposition 1.6 that

Theorem 1.7 (cf. [7]). The next equality holds:

$$
\operatorname{hol}(D, g)=(-1)^{\operatorname{Index}(D)} e^{-2 \pi i \xi_{g}}
$$

for any $g \in G$.

\section{AN EXAMPLE} by

Let $M$ be the non-singular hypersurface of degree $p \geq 2$ in $\mathbb{C P}^{n+1}$ defined

$$
z_{0}^{p}+z_{1}^{p}+\cdots+z_{n+1}^{p}=0
$$

where $\left[z_{0}: z_{1}: \cdots: z_{n+1}\right]$ is the homogeneous coordinate of $\mathbb{C P}^{n+1}$. Then the action

$$
g \cdot\left[z_{0}: z_{1}: \cdots: z_{n+1}\right]=\left[e^{2 \pi i / p} z_{0}: z_{1}: \cdots: z_{n+1}\right]
$$

defines an action of $\mathbb{Z}_{p}=\langle g\rangle$ on $M$ and the fixed point set of this action is the non-singular hypersurface of degree $p$ in $\mathbb{C P}^{n}=\left\{z_{0}=0\right\} \subset \mathbb{C P}^{n+1}$ defined by

$$
z_{1}^{p}+z_{2}^{p}+\cdots+z_{n+1}^{p}=0 .
$$

Let $D$ be the Dolbeault operator on $M$ which is a $\mathbb{Z}_{p}$-equivariant elliptic operator. Then it follows from the Atiyah-Bott-Singer fixed point formula (see, for example, [9]) that Index $(D)$ is equal to the $x^{n}$-coefficient of

$$
\left(\frac{x}{1-e^{-x}}\right)^{n+2}\left(\frac{1-e^{-p x}}{p x}\right) \in \mathbb{C}[[x]]
$$

multiplied by $p$ and that Index $\left(D, g^{k}\right)$ is equal to the $x^{n-1}$-coefficient of

$$
\left(\frac{x}{1-e^{-x}}\right)^{n+1}\left(\frac{1-e^{-p x}}{p x}\right) \frac{1}{1-e^{-x} e^{-2 \pi i k / p}} \in \mathbb{C}[[x]]
$$

multiplied by $p$.

Now, for example, consider the case of $n=2,3$. Then we can obtain Tables 1 and 2 only from direct computations using Theorem 1.4 and the fixed point formula above. 
$n=2$

\begin{tabular}{ccccccccccccccc}
\hline$p$ & 3 & 4 & 5 & 6 & 7 & 8 & 9 & 10 & 11 & 12 & 13 & 14 & 15 & 16 \\
\hline $\log ($ hol $)$ & 0 & $\frac{1}{4}$ & 0 & $\frac{3}{6}$ & 0 & $\frac{6}{8}$ & 0 & 0 & 0 & $\frac{3}{12}$ & 0 & $\frac{7}{14}$ & 0 & $\frac{12}{16}$ \\
\hline
\end{tabular}

$n=3$

TABLE 2

\begin{tabular}{ccccccccccccccc}
\hline$p$ & 3 & 4 & 5 & 6 & 7 & 8 & 9 & 10 & 11 & 12 & 13 & 14 & 15 & 16 \\
\hline $\log (\mathrm{hol})$ & 0 & 0 & $\frac{4}{5}$ & 0 & 0 & 0 & 0 & $\frac{8}{10}$ & 0 & 0 & 0 & 0 & $\frac{12}{15}$ & 0 \\
\hline
\end{tabular}

where $\log ($ hol $)$ denotes $\frac{1}{2 \pi i} \log \operatorname{hol}(D, g) \bmod \mathbb{Z}$.

Remark 2.1. If $\mathrm{c}_{1}(M)>0$ (namely, $p \leq n+1$ ), it follows from the Kodaira vanishing theorem that $\operatorname{coker} D=\{0\}$ and that $\operatorname{ker} D$ is equal to the 1-dimensional space of constant functions on $M$ on which $\mathbb{Z}_{p}$ acts trivially. Therefore it immediately follows that $\operatorname{hol}(D, g)=\operatorname{det}(D, g)=1$ and hence that $\log ($ hol $)=0$. This can also be proved from direct calculations similar as above using the Atiyah-Bott-Singer fixed point formula.

\section{APPENDIX}

Lemma 1. Let $A$ be an $(N \times N)$-matrix which satisfies $A^{p}=E$ for some positive integer $p$ where $E$ denotes the unit matrix. Then the next equality holds:

$$
\operatorname{det}(A)=\exp \frac{2 \pi i}{p} \sum_{k=1}^{p-1} \frac{1}{1-e^{-2 \pi i k / p}}\left\{N-\operatorname{tr}\left(A^{k}\right)\right\} .
$$

Proof. Let $e^{2 \pi i \lambda_{j} / p}(1 \leq j \leq N)$ be the eigenvalues of $A$ where $\lambda_{j}$ 's are integers such that $1 \leq \lambda_{j} \leq p$. Then the equality of the lemma is equivalent to the next equality:

$$
\lambda_{1}+\cdots+\lambda_{N}=\sum_{k=1}^{p-1} \frac{1}{1-e^{-2 \pi i k / p}} \sum_{j=1}^{N}\left(1-e^{2 \pi i \lambda_{j} k / p}\right) \quad \bmod . p .
$$

Therefore it suffices to show that

$$
\sum_{k=1}^{p-1} \frac{1-e^{2 \pi i k \lambda / p}}{1-e^{-2 \pi i k / p}}=\lambda \quad \bmod . p
$$

for any integer $\lambda$ such that $1 \leq \lambda \leq p$. Here the left-hand side of (1) is equal to $-\sum_{k=1}^{p-1} \sum_{\nu=1}^{\lambda} e^{2 \pi i k \nu / p}$ and hence (1) follows from the equality

$$
\sum_{k=1}^{p-1} e^{2 \pi i \nu k / p}=-1 \quad \bmod . p
$$

for any integer $\nu$. 
Lemma 2. Let $V$ be any finite-dimensional $\mathbb{Z}_{p}$-module and $g \in \mathbb{Z}_{p}$. Then we have

$$
\sum_{k=1}^{p} \operatorname{tr}\left(\left.g^{k}\right|_{V}\right)=0 \quad \text { mod.p. }
$$

Proof. This lemma follows from the equality

$$
\sum_{k=1}^{p} \alpha^{k}=0 \quad \bmod . p
$$

for any complex number $\alpha$ such that $\alpha^{p}=1$.

Lemma 3. The next equality holds:

$$
\frac{1}{2 p}=\frac{1}{2}-\frac{1}{p} \sum_{k=1}^{p-1} \frac{1}{1-e^{-2 \pi i k / p}} .
$$

Proof. This lemma follows from the equality

$$
\sum_{k=1}^{p-1} \frac{1}{1-e^{-2 \pi i k / p}}=\frac{p-1}{2} .
$$

\section{REFERENCES}

1. M. F. Atiyah and R. Bott, A Lefschetz fixed point formula for elliptic complexes I, Ann. of Matn. (2) 86 (1967), 374-407.

2. __ A Lefschetz fixed point formula for elliptic complexes II. Applications, Ann. of Math. (2) 88 (1968), 451-491.

3. M. F. Atiyah, V. K. Patodi, and I. M. Singer, Spectral asymmetry and Riemannian geometry I, Math. Proc. Cambridge Philos. Soc. 77 (1975), 43-69.

4. _ـ Spectral asymmetry and Riemannian geometry II, Math. Proc. Cambridge Philos. Soc. 78 (1975), 405-432.

5. M. F. Atiyah and I. M. Singer, The index of elliptic operators III, Ann. of Math. (2) 87 (1968), 546-604.

6. $ـ$ The index of elliptic operators IV, Ann. of Math. (2) 92 (1970), 119-138.

7. J. M. Bismut and D. S. Freed, The analysis of elliptic families II, Comm. Math. Phys. 107 (1986), 103-163.

8. H. Donnelly, Eta invariants for G-spaces, Indiana Univ. Math. J. 27 (1978), 889-918.

9. A. Hattori, Spin ${ }^{c}$-structures and $S^{1}$-actions, Invent. Math. 48 (1978), 7-31.

Tokyo University of Fisheries, 4-5-7 Kounan, Minato-kU, Tokyo 108, JAPAN

E-mail address: tsuboi@tokyo-u-fish.ac.jp 